Chapter 16

\title{
Surgical Therapy of Parkinson's Disease
}

\author{
Massimo Piacentino, Giacomo Beggio and \\ Lorenzo Volpin
}

Additional information is available at the end of the chapter

http://dx.doi.org/10.5772/62884

\begin{abstract}
The history of surgical treatment of Parkinson's disease (PD) covers more than 100 years. It started from lesional approach and evolved to the final deep brain stimulation (DBS) only in the 1990s. The aim of this treatment was to reduce clinical manifestation of PD and drug intake by acting directly on the altered motor pathways. The typical targets are represented by ventralis intermedius thalamic nucleus (VIM), internal globus pallidus nucleus (GPi), and subthalamic nucleus (STN) with more recent extension on other anatomicalstructuresaspedunculopontinenucleus(PPN).Patientsareselected according to CAPSIT protocol and undergo DBS when medical treatment has failed to effectively control the symptoms. Clinical benefits are represented by the reduction of "off" time and "on" time with dyskinesia. However, even DBS treatment is characterized by complications and side effects, as intracerebral hemorrhages, infections, ischemia, and seizures. The recent introduction of neuronavigation systems and the amelioration in neuroradiological imaging quality simplified preoperative DBS planning and consequently reduced surgical-related problems
\end{abstract}

Keywords: Deep brain stimulation, Parkinson surgical treatment, DBS target, surgical outcome, DBS complications

\section{Introduction}

The first experiences concerning surgical treatment of Parkinson's disease (PD) were made between the end of the nineteenth century and the beginning of twentieth century by Sir V. Horsely with surgery performed on cortical motor area. In the same period, Leriche et al. [1] attempted to improve rigidity and tremor by focusing on pyramidal tracts, in particular trying to interrupt abnormal motor signals by removing motor cortex, lesioning cervical spinal cord, 
or cerebral peduncle. Just before the World War II, the focus of surgical interest shifted to the basal ganglia with the work of Meyers on pallidofugal fibers and Russell, which completed a transventricular approach to remove the anterior two thirds of the caudate nucleus. However, the modern era can be traced by Spiegel [2] with the first stereotactic technique approaching globus pallidus (GPi). This author introduced the idea of a three-dimensional spatial target identification based on pneumoencephalography, ventriculography, and spatial relationship of targets in relation to a reference line connecting the foramen of Monro to the posterior commissure. In 1952, stereotactic thalamotomies were made in Freiburg, and between 1950 and 1960, surgical treatment of Parkinson was conducted in a quite homogeneous way by pallidotomy and thalamotomy with remarkable improvement of both tremor and rigidity. During the 1960s, the discovery of levodopa effect on PD symptoms induced a loss of interest on surgical treatment for almost three decades [3].

In the 1990s, the amelioration of diagnostic systems, with the introduction of computed tomography (CT), and the awareness of the inevitability of levodopa side effects gave a renewed strength to surgical approach to PD. However, Benabid and colleagues with chronic deep brain stimulation (DBS) introduced the real change of PD surgical therapy [4]. The first DBS target to be identified was ventral intermediate nucleus (Vim), followed later by subthalamic nucleus (STN) and internal segment of globus pallidus (GPi). In the following years, after the approval of US FDA to STN and GPi DBS (2002), this technique rapidly obtained the title of recognized therapy for advanced PD.

Recently, the introduction of magnetic resonance imaging (MRI) - Angio CT neuronavigation, intraoperative neurophysiologic evaluation and frameless systems gave rise to further improvements opening the doors to possible alternatives to the utilization of the classic stereotactic frames.

\section{Stereotactic systems}

In almost all neurosurgical centers, stereotactic surgery is conducted by the utilization of classic stereotactic frames in combination with either MRI or MRI and CT scan. This system is based on the determination of spatial relationship between an identified target into the brain contest and the frame rods visible on the MRI or CT images. Therefore, it is possible to determine the exact location of the target in relation to the Cartesian axes as well as the trajectories angles and the entry point. The computation of the coordinates and angles is performed by dedicated computer systems and more frequently by the neuronavigation devices available on the market. Finally, obtained coordinates and angle values are reported on specific millimetric scales of the stereotactic frame in order to have a chosen position of the electrodes on the entry point and along the planned trajectory to the target. During surgery, it is possible to perform $X$-ray control to confirm the exact position of the electrode in relation to the chosen target (Figure 1). 


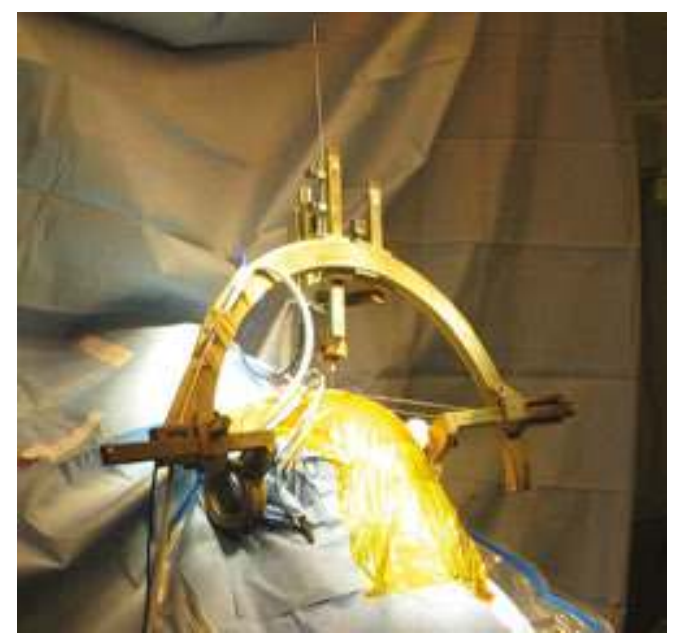

Figure 1. Stereotactic frame.

More recently, different companies have launched new frameless systems. This tools are based on the utilization of external fiducials (usually 6) fixed to the skull and consequently recorded on radiological studies (MRI and CT) acquired on the navigation system in order to reconstruct the entire head volume and report chosen targets, entry points, and trajectories. This new approach leaves the patient free from obliged and fixed position with less discomfort and fatigue. Moreover, these new systems do not differ in accuracy from classic stereotactic frame (Figures 2 and 3).

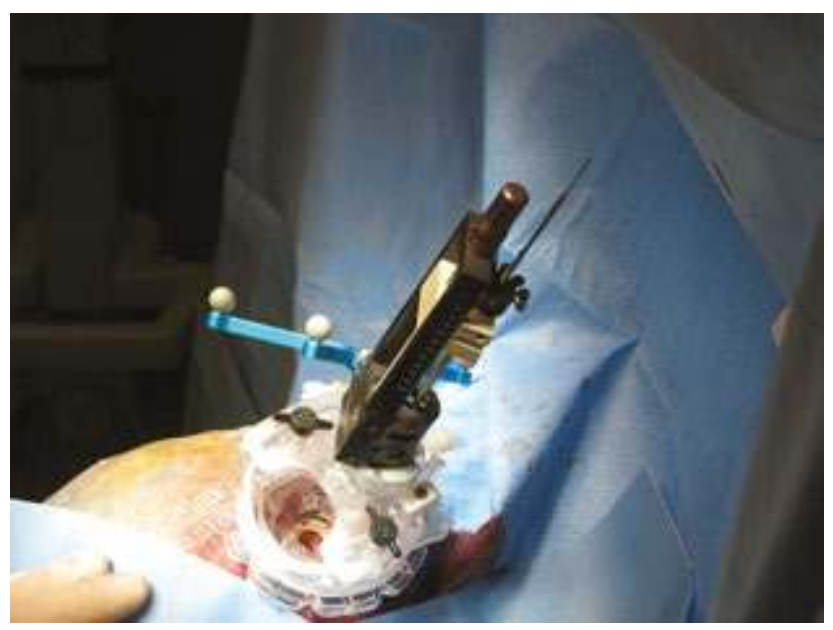

Figure 2. Frameless system. 


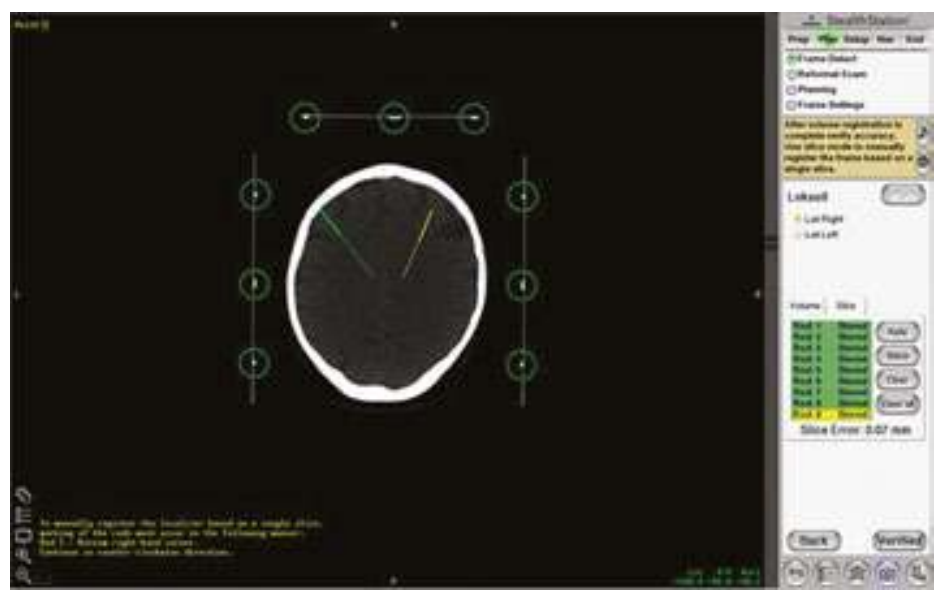

Figure 3. Fiducials image on neuronavigation system.

\section{Target identification}

Although in recent years, we assisted to a progressive improvement of technology, there are still strongholds that come from the past. In fact, neurosurgeons still consider the classical

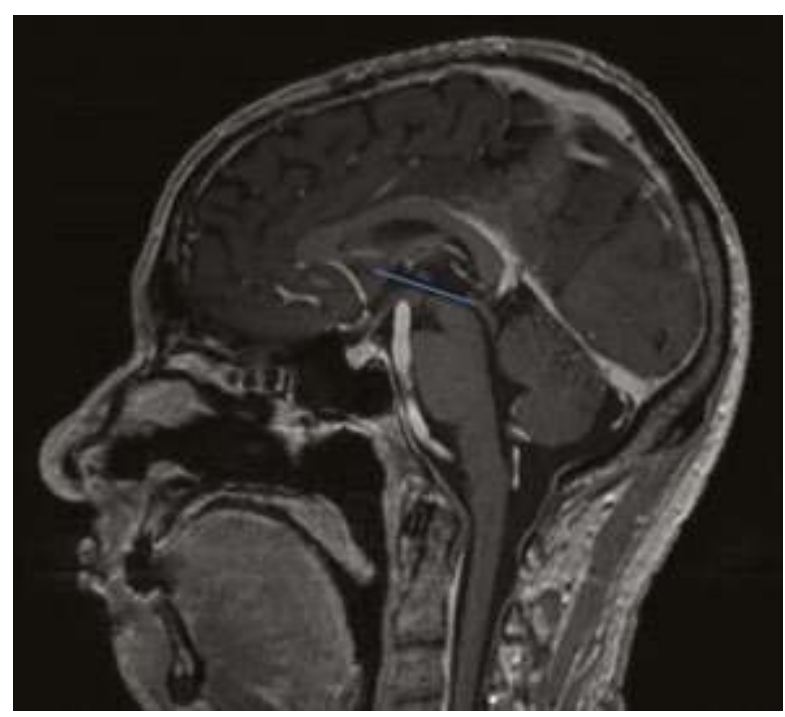

Figure 4. Intercommissural line marked in blue on Gadolinium T1-weighted sagittal MRI. 
Intercommissural Line (anterior commissure AC-posterior commissure PC line, AC-PC line, IC line) as a reference to determine stereotactic coordinates, which are consequently used as starting points to find specific anatomical targets (indirect targeting methodology) (Figure 4).

However, recent amelioration of MRI quality allowed a direct visualization of most of anatomical structures and a consequent easier direct surgical targeting (direct targeting methodology). Although this technical progress, it is always recommended to use both methods to adjust indirect final position to anatomical structures visible on MRI (Figure 5).

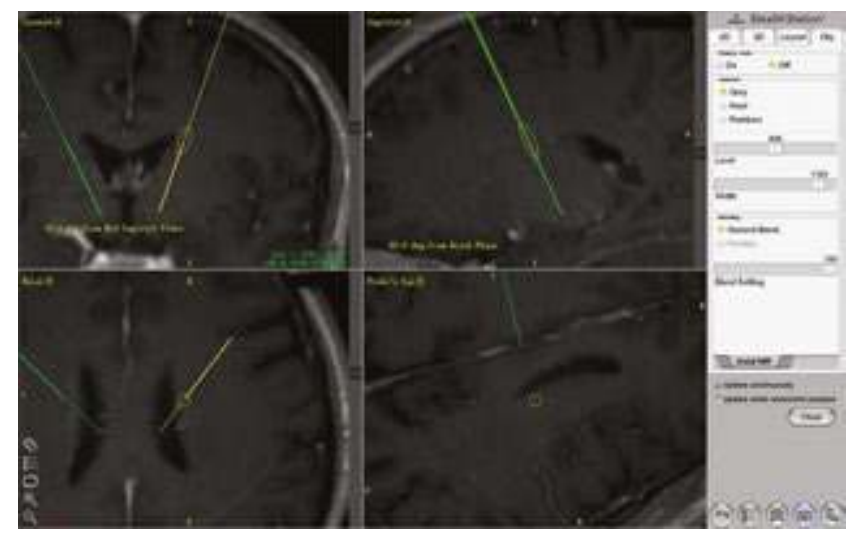

Figure 5. Trajectory planning on neuronavigation system.

The introduction of neuronavigation systems brought amelioration in pre-surgical determination of entry point and trajectory. Angio CT merged to Gadolinium T1-weighted MRI can better visualize vessels, bundles, and nuclei. Even if some authors do not consider strictly necessary to have both T1-weighted MRI and Angio CT for trajectories planning, sometimes the resolution of a single exam could not allow a complete and satisfactory vision in particular for small deep vessels (Figure 6).

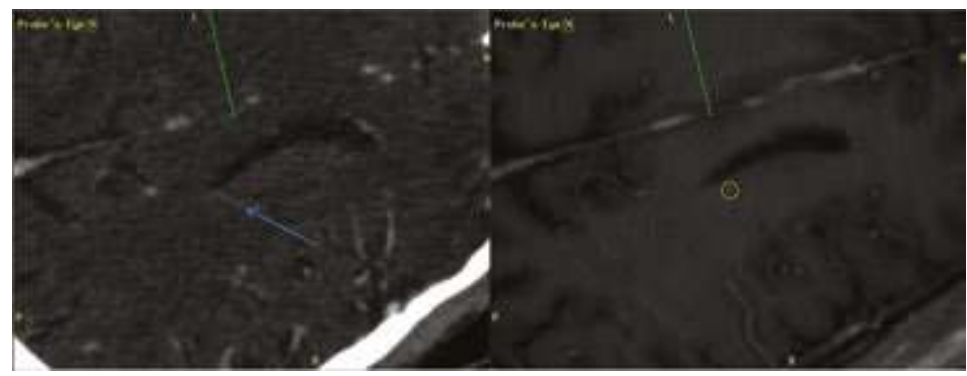

Figure 6. Confrontation between Angio CT and Gadolinium T1-weighted axial MRI; the arrow shows a vessel posterior to the trajectory only visible on Angio CT. 
Finally, it is important to remark the role of T2-weighted or inversion recovery sequences for the location of some anatomical structures, as STN or GPi, respectively, and proton density studies for direct determination of PPN.

Generally, implantation procedure is made with an awoken patient because of the necessity to stimulate the chosen target and avoid postoperative side effects. However, some authors reported experience of DBS in Parkinson's patients under general anesthesia considering secondary the neurophysiological aspect, and others just performed stimulation with macro electrode.

Once the electrodes have been positioned in the proper targets, the stimulation device (internal pulse generator, IPG) is hosted in a subclavicular subcutaneous pouch, and connections among IPG, extension, and intracerebral electrodes are performed under general anesthesia. An important consideration must be made about the number of electrode utilized during intraoperative recording that could vary from 1 to 5 . An important concern, in relation to this aspect, is in fact particularly linked to the necessity to find the best compromise between an efficient neurophysiologic test and the risk of hemorrhages.

Intraoperative electrical activity recording may give rise to a typical electrical pattern depending on the anatomical structure found along the electrode trajectory. Stimulation could also produce improvement of Parkinson's symptoms or induce different side effects giving important information about the exact targeting and possible correction to be made in relation to the obtained results.

\section{Surgical aspects}

In present times, deep brain stimulation (DBS) surgery can be performed with different systems that share however two common starting points. These are represented by the selection of PD patients and the choice of anatomical target.

\subsection{Patient selection}

The first attempt to standardize patient selection was developed in 1992 with the publication of CAPIT (core assessment program for intracerebral transplantation) that had the purpose of evaluate the outcome of patients selected for intracerebral transplantation of fetal dopamine neurons [5]. The evolution of this protocol, which included also patients treated by ablative and neurostimulation procedures, is represented by core assessment program for surgical interventional therapies (CAPSIT), which have been published in 1999 [6]. This protocol gained a growing importance during the last years and now represents the base for a correct selection of PD patients. In fact, it is mandatory to choose patients in which the clinical benefits of DBS overcome surgical risks connected to the procedure. Patients not only should be characterized by PD but also should be prepared in term of cognition, emotion, and social relations to the DBS procedure in order to maximize the treatment effect. 
CAPSIT-PD inclusion criteria are as follows: idiopathic Parkinson's disease; duration of illness $>5$ years; age between 35 and 70 years; improving UPDRS motor part of at least $40 \%$ at the L-Dopa test; persistence of severe disability at certain times of the day despite all the adopted therapeutic strategies; antiparkinsonian therapy stabilized during the month before the implant; absence of dementia (MMSE >24); absence of severe depression with suicidal ideations; absence of psychosis; MRI within normal limits without evidence of marked atrophy, or multiple abnormalities of vascular origin, or diseases interfering with the surgical procedure (e.g., tumors); no history of drug addiction; absence of disease or medication interfering with the coagulation.

These criteria describe three major groups of PD patients that could effectively benefit from DBS surgery. The first group is represented by patients affected by idiopathic PD with normal cognitive capacities that show motor fluctuations and dyskinesia despite optimal levodopa therapy. Patients with controlled PD with persistent medication-resistant tremor compose the second group. Patients who do not tolerate medical treatment with consequently poor symptoms control represent the third category of DBS candidates.

\subsection{DBS target identification}

Since Vim chronic stimulation has proven its efficacy only for tremor control, this nucleus is not considered as a useful target for PD patient [7]. For this reason, possible stimulation sites are chosen between internal globus pallidus (GPi) and subthalamic nucleus (STN) associated in certain cases to pedunculopontine nucleus (PPN) [8]. The difference between STN and GPi stimulation consists in a greater effect in controlling tremor by STN stimulation with a consistent reduction of therapy intake and a consequent better control of psychiatric side effects due to L-Dopa. Conversely, targeting of GPi leads to a major anti-dyskinetic effect but leaves pharmacological dosage unchanged with all possible consequences on the psychiatric condition. Nevertheless, even STN stimulation could produce psychiatric side effects concerning mood and behavioral troubles. Some authors consider the possibility to stimulate PPN in order to obtain an improvement in axial manifestations such as postural instability or gait dysfunction $[9,10]$.

\subsubsection{Targeting of internal globus pallidus (GPi)}

Internal globus pallidus nucleus could be identified using a direct or an indirect method. According to the scheme reported by Laitinen, indirect targeting of the GPi is obtained, 2-3 $\mathrm{mm}$ in front of the mid-AC-PC-point, 3-6 mm caudal to intercommissural (IC) plane, and 20$21 \mathrm{~mm}$ lateral to the AC-PC line.

The planning requires the utilization of T2-weighted or inversion recovery (IR) MRI to locate the intercommissural line and draw the coordinate projection. T1-contrast images are also used to identify sulci gyri and vessels for the identification of safe trajectories avoiding vessels. GPi can be found in the medial part of the globus pallidus, medial to the lamina medullaris interna and lateral to the genu/posterior limb of internal capsule on the axial plane. Coronal plane can be used to obtain a better definition of GPi's relationship with optic radiations. Once the target 
is reached, intraoperative recording shows a specific electrical pattern consisting in a high frequency activity with 40 spikes/s of tonic firing [11]. Visual spots are conversely evocated when the electrode trespasses on the optic radiations. This sign is important to determine the lower boundary of the GPI and to avoid visual impairments once the device is completely implanted and programmed (Figures 7 and 8 ).

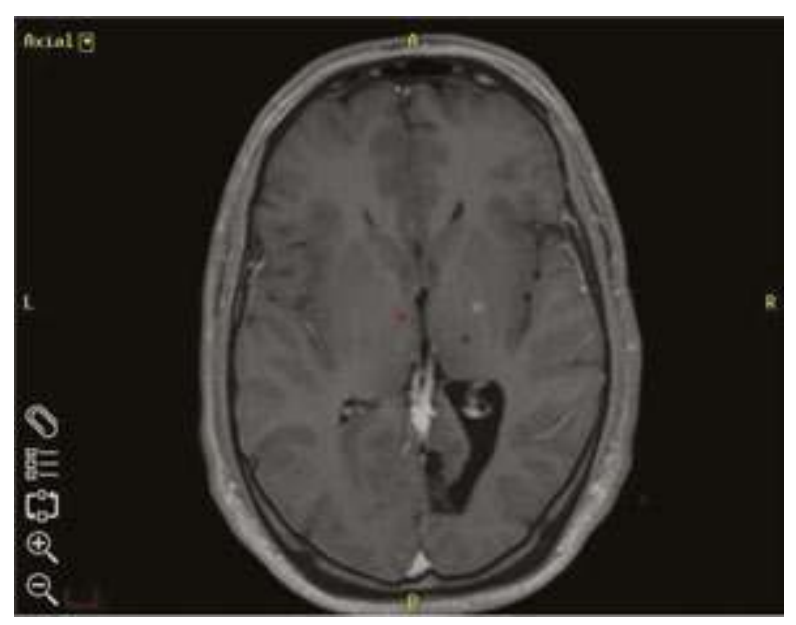

Figure 7. Electrode final position in GPI.

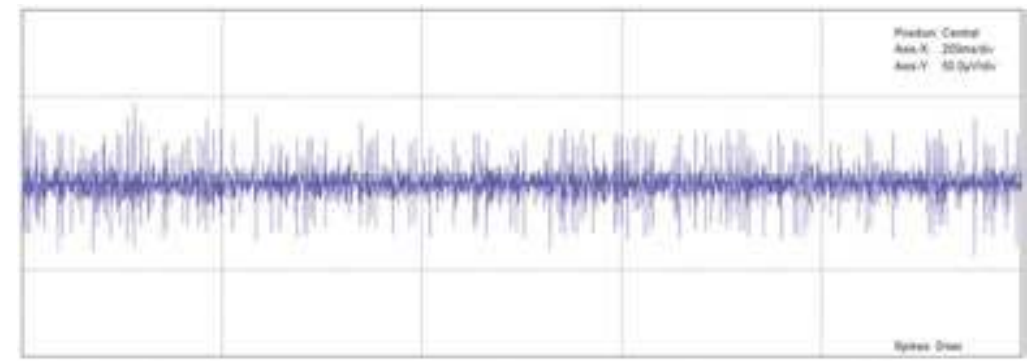

Figure 8. Intraoperative GPI recording.

\subsubsection{Targeting of subthalamic nucleus (STN)}

Indirect coordinates of the STN are reported as 3-4 mm posterior to mid-AC-PC-point, 3-5 $\mathrm{mm}$ below the AC-PC line, 11-12 mm lateral to AC-PC line. MRI images and in particular T2weighted acquisitions may give a clear vision of the subthalamic nucleus as well as its anatomical relationship with other structures such as red nucleus, Forel's fields, and zona incerta (Figure 9). 


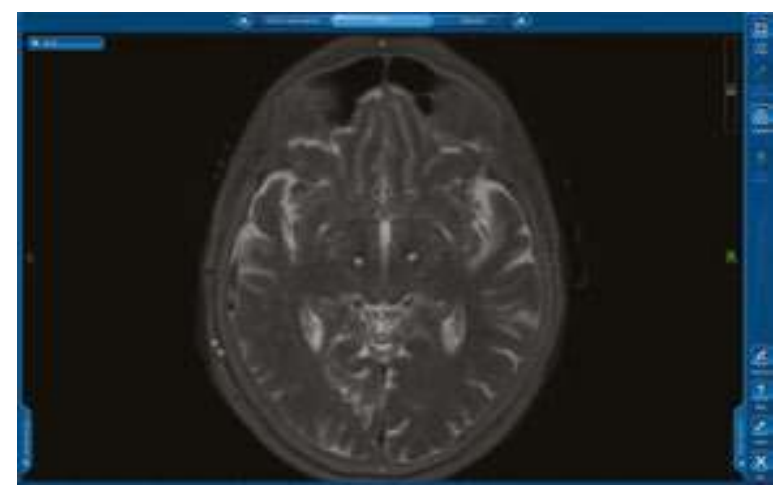

Figure 9. Final electrode position showed on CT merged to T2-axial MRI.

Neurophysiological intraoperative monitoring could give important information not only evidencing the typical electrical pattern of STN (STN neurons are characterized by a mean firing rate of $42.30 \pm 22.00$ spikes/sec (mean $\pm S D$ ); the STN cells exhibited irregular or bursty discharge pattern) but also inducing improvement of Parkinson's symptoms or even side effects as paresthesias, muscular spasticity, diplopia, speech difficulties, and neurovegetative alterations [11, 12]. Therefore, neurophysiological intraoperative tests play an important role for the determination of the final electrode position, in order to maximize the benefit and avoid side effects lied to a possible brain shift secondary to CSF leakage and air penetration. During target planning, an important step is to avoid the anterior part of the STN, which is involved not only in extrapyramidal pathways but also in limbic circuits. Effectively, a stimulus that involves the anterior part of STN may cause behavioral changes, depression,

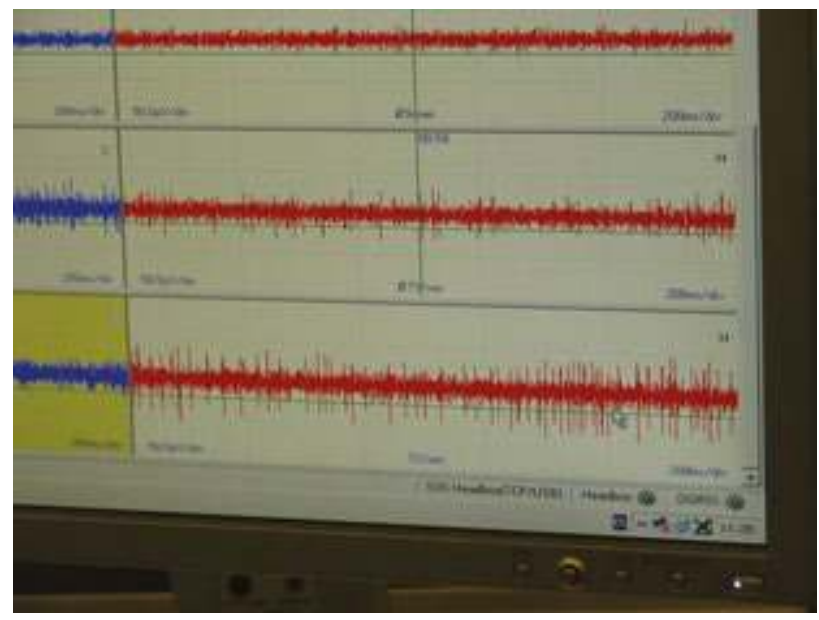

Figure 10. Intraoperative recording of STN electrical activity. 
maniac episodes, and other psychiatric complications. The diffusion of the stimulus to the substantia nigra may also give rise to psychiatric problems such as major depression and hypomania [13] (Figure 10).

\subsubsection{Targeting ventralis intermedius thalamic nucleus (VIM)}

VIM stimulation is used in patients affected by tremor (parkinsonian or essential), and its indirect target is $25-50 \%$ of AC-PC length from PC at IC plane, $1 / 2$ (third ventricular width) $+11.5 \mathrm{~mm}$. These coordinates are actually reported on MRI images, where the nucleus is not normally visible on 3 Tesla MRI. Recorded electrical pattern has no specific features and does not differ from other sites within the thalamus. Conversely, the stimulation test can be very useful not only in reducing or abolishing tremor immediately but also in defining the boundaries of the VIM by evocating paresthesia or other sensitive symptoms secondary to the stimulation of the VPL nucleus [14] (Figures 11 and 12).

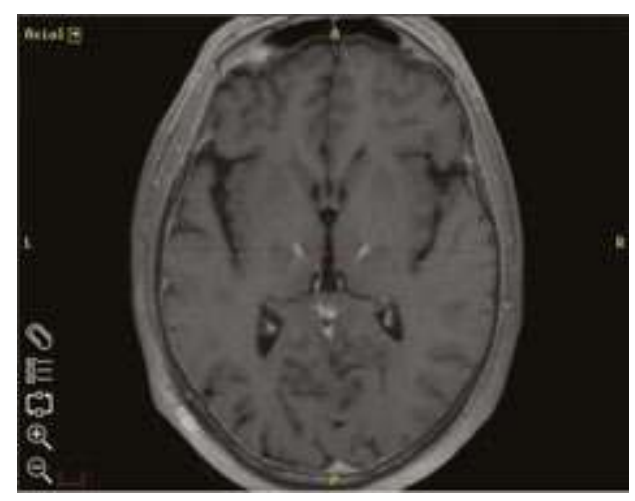

Figure 11. Final electrodes position in VIM showed on neuronavigation system.

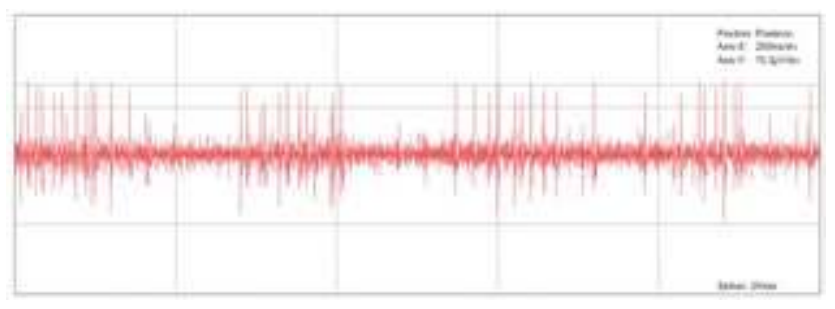

Figure 12. Intraoperative VIM recording showing typical low-frequency spikes synchronized with tremor. 


\subsubsection{Targeting of pedunculopontine nucleus (PPN)}

The stimulation of this nucleus is utilized to reduce or abolish gait disturbances and freezing. The indirect localization may be obtained considering a line passing anterior to the floor of the fourth ventricle on a line running along the pontomesencephalic longitudinal axis (Figure 13).

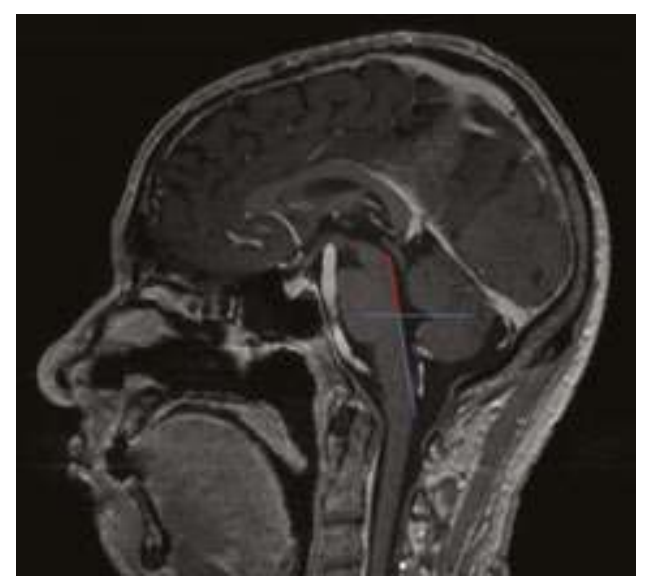

Figure 13. Gadolinium T1-sagittal MRI showing indirect localization of PPN; the red tract above the fastigial line indicates the location of PPN.

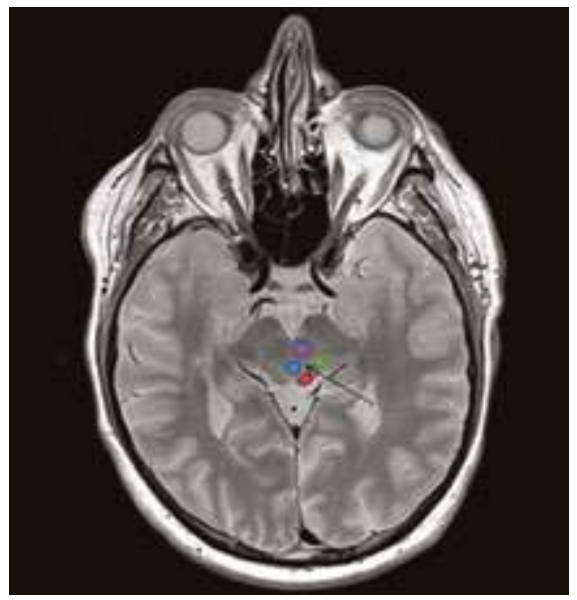

Figure 14. Proton density axial MRI; the arrow shows the PPN location; the blue area indicates the periaqueductal grey matter, the violet circle indicates the red nucleus/decussation of anterior cerebellar peduncle, the green oval indicates 
lateral lemniscus and the red area indicates the medial lemniscus/spinothalamic tract; on the left side of the image, the anatomical structures are visible in the real grey scale.

This line is crossed by an orthogonal line traced through the fastigium in order to identify a superior and inferior component of the previous identified line. Using this reference system, the PPN is identified $6 \mathrm{~mm}$ lateral and $4 \mathrm{~mm}$ anterior to the pontomesencephalic/fastigial lines, $16 \mathrm{~mm}$ above the crossing point between the base point fastigial line. The direct localization may be obtained by MRI identification using proton density images. The target is identified on an axial slice passing through the inferior colliculus in an area localized among the decussation of anterior cerebellar peduncle anteriorly, medial lemniscus anterolaterally, lateral lemniscus and spinothalamic tracts posterolaterally, periaqueductal gray matter and middle longitudinal fasciculus medially (Figure 14).

Recording of this area does not give specific information, while stimulation may be helpful in determining the exact position of the electrode contacts in relation to the absence of evoked side effects. The diffusion of the stimulus to the close structures could cause sensitive disturbances in case of stimulation of the lemniscal/spinothalamic bundles, hearing and vertigo stimulating medial lemniscus, diplopia secondary to the activation of third cranial nerve sub-nuclei, neurovegetative imbalance for the stimulation of central tegmental neurons [15].

\section{IPG programming}

IPG programming takes place after a first period of postoperative rest in order to focalize better the real effect of DBS without the bias related to the surgical damage secondary to leads introduction. The optimization of IPG stimulation pattern could necessitate several months, usually from 4 to 6 . Each electrode can range from four to eight contacts that could be spaced 0.5 or $1.5 \mathrm{~mm}$, and the stimulation could be made in a monopolar or bipolar way. The chosen configuration should be characterized by the best clinical effect with the smallest amount of side effects, which could derive from the extension of electrical stimulation to neighboring anatomical structures.

Once IPG setting is completed, the attention is focalized on stimulation amplitude, which is usually increased gradually in order to obtain a reduction in antiparkinsonian drug intake while preserving clinical wellness [16].

Finally, it is important to pay attention to frequency stimulation. Since the beginning of DBS treatment, high-frequency stimulation (above $130 \mathrm{~Hz}$ ) has been considered the base of clinical improvement. However, more recently several authors showed the great potential of intermediate frequencies $(60-80 \mathrm{~Hz})$ in particular on freezing and gait even if the clinical benefit could be only temporary $[17,18]$. 


\section{Outcomes}

Recent randomized controlled clinical trials on GPi and STN DBS showed that these treatments are superior to PD medical management alone [19].

As reported in a review by Duker et al. [16] PD DBS patients could obtain almost 4.4-4.6 h per day of "on" time without dyskinesia and a reduction of 1-2.6 h per day of "on" time with dyskinesia and $2.4-4.2 \mathrm{~h}$ per day of "off" time.

Since it has been proved that STN and GPi DBS are characterized by the absence of difference in motor function, the selection of the anatomical target should rely on patient peculiarities and surgeon preference [10].

\section{Timing of DBS in Parkinson patients}

In the last years, several authors focused their attention on DBS timing, reporting the hypothesis of a larger and more lasting amelioration of PD symptoms with early surgical treatment. As reported by Schuepbach et al. [20] in 2013, an earlier DBS could in fact improve patient quality of life better than medication alone even only after 2 years of PD. Conversely till nowadays, DBS solution was reserved usually for patients that had a minimum history of 5 years of disease and motor symptoms that did not improved with optimized medical therapy [6]. More studies are, however, necessary in order to further verify this hypothesis and to clarify long-term efficacy of this early surgical approach [16].

\section{Complications and side effects}

Although DBS is a relatively safe and effective procedure, complications and side effects represent a rare but unavoidable constant in this surgical treatment. As reported by recent literature reviews, adverse effects can be divided into 3 time-related categories: intraoperative, perioperative ( $<2$ weeks), and long-term postoperative complications ( $>2$ weeks). Intraoperative adverse events are represented by intracerebral $(\mathrm{ICH})$ and intraventricular (IVH) hemorrhage, acute perilesional edema, cortical/subcortical ischemic infarction, vasovagal response and hypotension, confusion, anxiety, seizure, arrhythmia, and aborted procedure. Perioperative complications, which arise during the first 2-week postop, are headache, hemiparesis, confusion, agitation, respiratory distress, seizure, hallucinations, somnolence, and falls. Conversely, long-term postoperative complications are represented by wound complications (infection, skin erosion, wound dehiscence), hardware complications (lead fracture/malposition/migration/malfunction, flipped IPG, malpositioned/uncomfortable/ malfunctioning internal pulse generator (IPG), lead extension malfunction/fracture), and satisfaction-related complications (loss of system efficacy over time, decreased efficacy over time) [21]. 
The recent improvement in pre-surgical target planning, with the introduction of neuronavigation systems, and the quality improvement of neuroradiological imaging are decreasing the rate of intracranial hematomas connected to electrodes placement. However, this complication remains possible with all consequent damages ranging from minor asymptomatic bleedings to huge hemorrhages with fatal consequences. Medical treatment is based on administration of mannitol or steroids, depending on the clinical and radiological characteristics of the hematoma, and could be associated to surgical cloth evacuation.

Infections represent another important DBS complication. Infection sites are frequently located at the level of the pouch created for the device, more rarely along the extension cables (usually due to a propagation from the infected device pouch) or on the intracranial extension of the electrodes, with consequent meningitis, subdural empyema, or intracerebral abscess. Antibiogram, specific antibiotic treatment, and removal of the infected components are mandatory except in case of superficial skin infection with no propagation of the components of the implanted system.

Wound dehiscence is usually caused by cutaneous decubitus of stimulation device. This complication is often related to a subsequent infection frequently caused by saprophytes cutaneous germs. It requires wound reopening, tissue debridement, and change or increase of the pouch volume.

Dislocation may be possible due to the relative tenderness of the electrode tip that could deviate from the chosen target. In order to reduce this possibility, it is advisable to use long cannulas to guide the electrode to the target.

Sometimes the electrode may move upward or downward from the target. This problem is often related to a blockage failure and requires the removal of the electrode and a new surgical positioning.

The brain is normally contained in a liquid environment formed by cerebrospinal fluid (CSF). During surgery, a certain volume of CSF leaks trough the surgical opening giving rise to a change in spatial relationship between the brain and the skull. In order to minimize, this problem many authors planned different solutions but a minimal amount of brain shift is still unavoidable. Obviously, a careful and minimal opening of the skull and meninges and the neurophysiological intraoperative recording may minimize this effect without affecting the surgical outcome (Figure 15). 


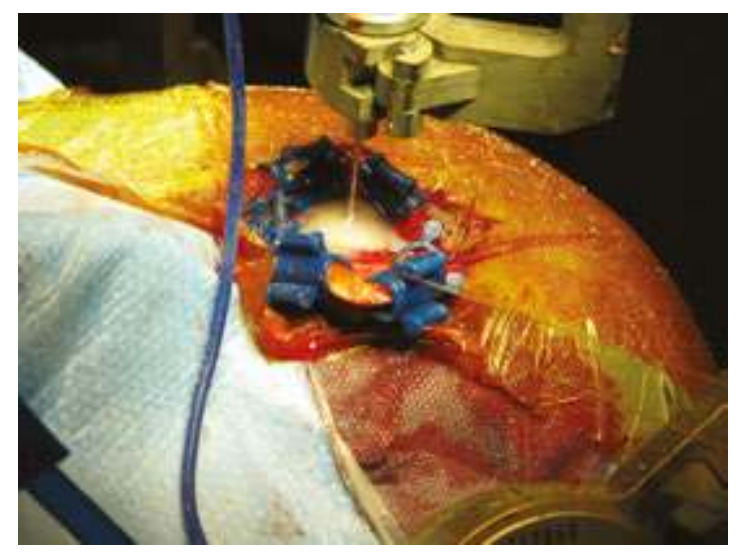

Figure 15. Fibrin glue and hemostatic sponge used as a plug to minimize CSF leakage.

A careful planning and intraoperative neurophysiological evaluation is usually sufficient to avoid side effects. However, complications as brain shift, electrode dislocation, or difficulty to evidence the evoked symptoms due to poor cooperation and reliability of the patient could cause postoperative problems. Propagation of the stimulus outside the planned target could induce pyramidal symptoms occurring by to the internal capsule, eye movement imbalance, appraisal, or worsening of dysarthria, psychiatric alteration, paresthesia, or skin numbness (this last complication normally disappears rapidly thanks to neuronal adaptation). Moreover, attention must be paid during trajectory planning to avoid caudate nucleus, in order to prevent cognitive deterioration.

\section{Author details}

Massimo Piacentino*, Giacomo Beggio and Lorenzo Volpin

*Address all correspondence to: massimo.piacentino@ulssvicenza.it

Department of Neurosurgery, San Bortolo Hospital, Vicenza, Italy

\section{References}

[1] Leriche R. Ueber Chirurgischen Eingriff bei Parkinson's scher Krankheit. Neurol Zeitblaetter. 1912;13:1093-1096.

[2] Spiegel EA, Wycis HT, Marks M, Lee AJ. Stereotaxic apparatus for operations on the human brain. Science. 1947;106(2754):349-350. 
[3] Cotzias GC, Van Woert MH, Schiffer LM. Aromatic amino acids and modification of parkinsonism. N Engl J Med. 1967;276(7):374-379.

[4] Benabid AL, Pollak P, Louveau A, Henry S, De Rougemont J. Combined (thalamotomy and stimulation) stereotactic surgery of the VIM thalamic nucleus for bilateral Parkinson disease. Appl Neurophysiol. 1987;50ca(1-6):344-346.

[5] Langston JW, Widner H, Goetz CG, Brooks D, Fahn S, Freeman T, et al. Core assessment program for intracerebral transplantations (CAPIT). Mov Disord. 1992;7(1):2-13.

[6] Defer GL, Widner H, Marie RM, Remy P, Levivier M. Core assessment program for surgical interventional therapies in Parkinson's disease (CAPSIT-PD). Mov Disord. 1999;14:572-584.

[7] Rehncrona S, Johnels B, Widner H, Törnqvist A-L, Hariz M, Sydow O. Long-term efficacy of thalamic deep brain stimulation for tremor: double-blind assessments. Mov Disord. 2003;18(2):163-170.

[8] Odekerken VJ, Boel JA, Schmand BA, de Haan RJ, Figee M, van den Munckhof P, et al. GPi vs STN deep brain stimulation for Parkinson disease: three-year follow-up. Neurology. 2016; 86(8):755-61. doi:10.1212/WNL.0000000000002401

[9] Yousif N, Bhatt H, Bain PG, Nandi D, Seemungal BM. The effect of pedunculopontine nucleus deep brain stimulation on postural sway and vestibular perception. Eur J Neurol. 2016; 23(3):668-70. doi:10.1111/ene.12947

[10] Follett KA, Torres-Russotto D. Deep brain stimulation of globus pallidus interna, subthalamic nucleus, and pedunculopontine nucleus for Parkinson's disease: which target? Parkinsonism Relat Disord. 2012;18(Suppl 1):S165-7. doi:10.1016/S13538020(11)70051-7

[11] Bour LI, Contarino MF, Foncke EM, de Bie RM, van den Munckhof P, Speelman JD, et al. Long-term experience with intraoperative microrecording during DBS neurosurgery in STN and GPi. Acta Neurochir (Wien). 2010;152(12):2069-77. doi:10.1007/s00701010-0835-y.

[12] Benazzouz A, Breit S, Koudsie A, Pollak P, Krack P, Benabid AL. Intraoperative microrecordings of the subthalamic nucleus in Parkinson's disease. Mov Disord. 2002;17(Suppl 3):S145-9.

[13] Ulla M, Thobois S, Llorca PM, Derost P, Lemaire JJ, Chereau-Boudet I, et al. Contact dependent reproducible hypomania induced by deep brain stimulation in Parkinson's disease: clinical, anatomical and functional imaging study. J Neurol Neurosurg Psychiatry. 2011;82(6):607-14. doi:10.1136/jnnp.2009.199323.

[14] Huss DS, Dallapiazza RF, Shah BB, Harrison MB, Diamond J, Elias WJ. Functional assessment and quality of life in essential tremor with bilateral or unilateral DBS and focused ultrasound thalamotomy. Mov Disord. 2015;30(14):1937-43. doi:10.1002/mds. 26455. 
[15] Golestanirad L, Elahi B, Graham SJ, Das S, Wald LL. Efficacy and safety of pedunculopontine nuclei (PPN) deep brain stimulation in the treatment of gait disorders: a metaanalysis of clinical studies. Can J Neurol Sci. 2016;43(1):120-6. doi:10.1017/cjn.2015.318

[16] Duker AP, Espay AJ. Surgical treatment of Parkinson disease: past, present, and future. Neurol Clin. 2013;31(3):799-808. doi:10.1016/j.ncl.2013.03.007.

[17] Moreau C, Defebvre L, Destée A, Bleuse S, Clement F, Blatt JL, et al. STN-DBS frequency effects on freezing of gait in advanced Parkinson disease. Neurology. 2008;71(2):80-84.

[18] Ricchi V, Zibetti M, Angrisano S, Merola A, Arduino N, Artusi CA, et al. Transient effects of $80 \mathrm{~Hz}$ stimulation on gait in STN DBS treated PD patients: a 15 months followup study. Brain Stimul. 2012;5(3):388-392.

[19] Williams A, Gill S, Varma T, Jenkinson C, Quinn N, Mitchell R, et al. Deep brain stimulation plus best medical therapy versus best medical therapy alone for advanced Parkinson's disease (PD SURG trial): a randomised, open-label trial. Lancet Neurol. 2010;9(6):581-591.

[20] Schuepbach WMM, Rau J, Knudsen K, Volkmann J, Krack P, Timmermann L, et al. Neurostimulation for Parkinson's disease with early motor complications. N Engl J Med. 2013;368(7):610-622.

[21] Fenoy AJ, Simpson RK Jr. Risks of common complications in deep brain stimulation surgery: management and avoidance. J Neurosurg. 2014;120(1):132-9. doi: 10.3171/2013.10.JNS131225. 
